\title{
ESTIMATION DE LA POLLUTION PAR METAUX LOURDS DANS LA SOMME PAR L'ANALYSE DES BRYOPHYTES AQUATIQUES
}

\author{
par A. EMPAIN (*)
}

\begin{abstract}
RESUME
L'intérêt des bioindicateurs fixés à cycle de vie long réside dans leur indépendance marquée vis-à-vis des saisons: ils intègrent les grandes variations des paramètres physico-chimiques sur une durée de temps appréciable et procurent une estimation stable et sensible de l'impact des multiples pollutions sur l'écosystème aquatique. Les bryophytes aquatiques seront utilisés ici pour leur pouvoir intégrateur et accumulateur des métaux lourds.
\end{abstract}

\section{INTRODUCTION}

Les peuplements de bryophytes aquatiques du cours belge de la Sambre et de la Meuse, ainsi que de la Somme (NW de la France) ont été étudiés en relation avec les caractéristiques physico-chimiques du milieu. Ces organismes présentent un intérêt certain pour l'estimation des pollutions en général (cf. EMPAIN, 1973, 1974 ; EMPAIN et LAMBINON, 1975), bien que la méthode d'estimation biologique de la qualité des eaux basée sur l'analyse du peuplement bryophytique ait une portée moins générale que celle prenant en considération la végétation algale. II est néanmoins certaines circonstances où les bryophytes se révèlent des *bioindicateurs - extraordinairement précieux. C'est le cas notamment en ce qui concerne les pollutions radioactives (cf. e.a. KIRCHMANN et LAMBINON, 1973), où de tous les animaux et végétaux testés, les bryophytes aquatiques manifestent le pouvoir accumulateur le plus marqué vis-à-vis des radionuclides considérés. De façon similaire, les bryophytes aquatiques présentent un intérêt particulier pour l'étude des pollutions par métaux lourds; c'est de cet aspect qu'il sera question ici.

(*) Aspirant du FNRS. Université de Liège, Département de Botanique, Sart Tilman, B-4000 Liège, Belgique. 
Fixés aux berges et aux ouvrages d'art, ces végétaux intègrent les variations physico-chimiques sur une période très longue, car ils restent bien développés toute l'année; ils subissent l'impact de toute pollution ponctuelle, même rare. De plus, la biomasse qu'ils représentent est en général amplement suffisante pour y réaliser des dosages de métaux lourds. Ces analyses contrastent donc avec celles effectuées directement dans l'eau, qui montrent de grandes variations d'une série de mesures à une autre, et où la probabilité de rencontrer un pic important est bien entendu faible; en outre, comme la tranche d'eau contaminée dérive dans l'espace, le profil instantané que l'on observe présente des irrégularités que l'on ne peut expliquer que par le déplacement vers l'aval d'une pollution ponctuelle.

\section{MATERIEL ET METHODES}

Les bryophytes analysés ici sont tous des mousses aquatiques, ou du moins se trouvant la majeure partie du temps immergées (EMPAIN. 1974). Les espèces les plus communes ont été retenues : Platyhypnidium riparioides (Heaw.) Dix. (= Rhynchostegium rusciforme B.S.G.), Cinclidotus nigricans (Brid.) Wijk et Marg. et Fontinalis antipyretica L. ex Hedw; à titre de comparaison Octodiceras fontanum (La Pyl.) Lindb. et Fissidens crassipes (Wils.) B.S.G. ont été récoltés

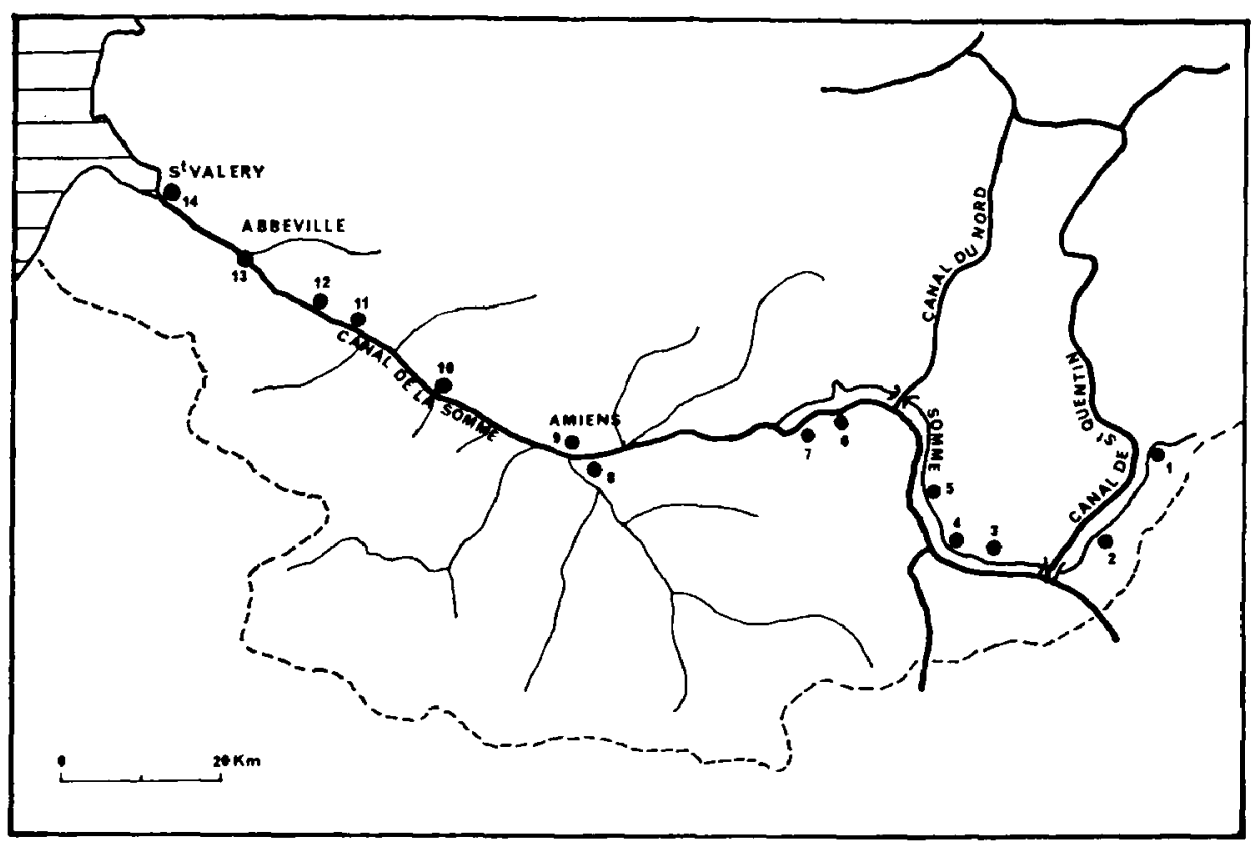

Figure 1: Répartition des stations de récoltes le long de la Somme.

1. Morcourt

2. Séraucourt

3. Ham

4. Offoy

5. Epénancourt

6. Frise

7. Cappy
8. Daours

9. Amiens

10. Picquigny

11. Long

12. Pont-Rémy

13. Abbeville

14. Saint-Valéry 
dans l'écluse d'Amiens. Montrant une résistance variable aux pollutions, ces espèces ne sont donc pas toutes représentées dans chaque station; elles sont même totalement absentes à Séraucourt (Fig. 1).

Les échantillons sont toujours recueillis en aval des déversoirs ou des écluses, nettoyés dans l'eau de la rivière et mis en sac de papier. Au laboratoire, ils sont lavés soigneusement à l'eau désionisée, séchés à $40^{\circ} \mathrm{C}$ et minéralisés par voie humide en présence de $\mathrm{HNO}_{3}$ et $\mathrm{HClO}_{4}$ à chaud. Les dosages en absorption atomique, comme la minéralisation, s'effectuent suivant les prescriptions fournies par PINTA (1971).

\section{RESULTATS DES ANALYSES}

Tableau 1 : Concentration des différents éléments dans les bryophytes analysés.

\begin{tabular}{|c|c|c|c|c|c|c|c|c|c|}
\hline \multirow[b]{2}{*}{ Espèces } & \multirow[b]{2}{*}{ Stations } & $\mathrm{Cd}$ & Co & $\mathrm{Cr}$ & $\mathrm{Cu}$ & $\mathrm{Fe}$ & $M n$ & $\mathrm{Ni}$ & $\mathrm{Pb}$ \\
\hline & & \multicolumn{2}{|c|}{$\begin{array}{r}\mathrm{mg} / \mathrm{kg} \text { de } \\
\mathrm{sec}\end{array}$} & poid & & $\times 10^{3}$ & $\times 10^{3}$ & & \\
\hline \multirow{11}{*}{$\begin{array}{l}\text { Platyhypnidium } \\
\text { riparioides }\end{array}$} & Morcourt & 0.1 & 8 & 7 & 42 & 2.1 & 0.2 & $<20$ & 45 \\
\hline & Ham & 0. & 36 & 16 & 2 & 9.0 & 21.6 & 460 & 85 \\
\hline & Offoy & 0.6 & 94 & 16 & 0 & & 38.8 & 700 & 70 \\
\hline & Epénancourt & 1.4 & 5 & 13 & & 4 & 0.4 & 80 & 70 \\
\hline & & 2.2 & 22 & 12 & 1 & 6. & & 50 & 30 \\
\hline & & 1.3 & 9 & 16 & 1 & 7. & 0.6 & 50 & 50 \\
\hline & Daol & 2.3 & 18 & 30 & 12 & 15. & 1.2 & 100 & 130 \\
\hline & Picq & 12.2 & 48 & 70 & 6 & 5 & 10.9 & 170 & 130 \\
\hline & & 6.2 & 13 & 53 & 6 & 6. & 13.0 & $<20$ & 70 \\
\hline & & 2.8 & 7 & 18 & 25 & 1.8 & 0.5 & $<20$ & 2 \\
\hline & & 4.0 & 7 & 25 & 6 & 3. & 0.3 & 80 & 85 \\
\hline \multirow{6}{*}{$\begin{array}{l}\text { Cinclidotus } \\
\text { nigricans }\end{array}$} & & 1.3 & 30 & 11 & 15 & 7. & 6.7 & 100 & 180 \\
\hline & & 1.0 & 32 & 7 & 27 & 7. & 6.9 & 100 & 150 \\
\hline & 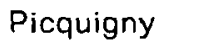 & 3.1 & 21 & 49 & 39 & 4. & 2.3 & 30 & 135 \\
\hline & & 4.9 & 28 & 48 & 42 & 4 & 4. & 60 & 125 \\
\hline & & 3.8 & 22 & 40 & 6 & 4. & 2.4 & 30 & 110 \\
\hline & ery & 1.4 & 9 & 16 & 42 & 2.2 & 1.0 & $<20$ & 55 \\
\hline \multirow{5}{*}{$\begin{array}{l}\text { Fontinalis } \\
\text { antipyretica }\end{array}$} & Daours & 1.9 & 48 & 15 & 15 & 5.4 & 25.0 & 420 & 40 \\
\hline & & 4.3 & 21 & 33 & 39 & 2. & 4.3 & 80 & 190 \\
\hline & -Rémy & 2.3 & 7 & 18 & 2 & 1 & 1.2 & $<20$ & 5 \\
\hline & eville & 2.4 & 15 & 15 & 49 & 1.6 & 1.9 & $<20$ & 75 \\
\hline & Saint-Valéry & 5.2 & 63 & 31 & 77 & 2.4 & 9.0 & 40 & 95 \\
\hline \multirow{3}{*}{$\begin{array}{l}\text { Octodiceras } \\
\text { fontanum } \\
\text { Fissidens } \\
\text { crassipes }\end{array}$} & Amiens & 1.9 & 44 & 8 & 29 & 10.2 & 12.2 & 30 & 210 \\
\hline & & & & & & & & & \\
\hline & Amiens & 1.9 & 26 & 10 & 25 & 6.3 & 5.9 & 80 & 90 \\
\hline \multicolumn{2}{|c|}{ Erreur de mesure : \pm} & 0.5 & 3 & 1.5 & 1.2 & 0.3 & 0.2 & 20 & $\overline{12.5}$ \\
\hline
\end{tabular}

Le tableau 1 rassemble les résultats des analyses des huit éléments considérés dans les espèces récoltees. L'examen de ces données et leur confrontation, à titre comparatif, avec celles inédites, obtenues pour la Sambre et la Meuse belges, permettent de dégager les principales considérations suivantes : 
1. Pour le cuivre: trois zones de concentrations un peu èlevées se rencontrent : près de la source (à Morcourt), à Epénancourt, et surtout en aval d'Amiens, où les plus fortes concentrations sont observées. Cependant, celles-ci restent de loin inférieures à celles de la haute Sambre belge.

2. La contamination par le cadmium, presque nulle au départ, augmente lentement et présente un pic en aval d'Amiens; ensuite, les valeurs diminuent rapidement vers l'aval.

3. Du même type que celui du cadmium, le profil du chrome souligne une contamination en aval d'Amiens, qui dépasse celle de la basse Meuse belge.

4. Des concentrations importantes, mais inférieures à celles de la Sambre et de la Meuse belges, en plomb et en fer, s'observent dès l'amont d'Amiens, avec en plus un pic de moindre importance dans la région d'Offoy.

5. Le cobalt, le nickel et le manganèse présentent un profil très comparable dans la Somme : un pic très important au niveau d'Offoy, suivi d'une décroissance rapide, puis d'une contamination progressive en aval d'Amiens. Les maxima observés dépassent ceux de la Sambre et de la Meuse belges: de cinq fois pour le nickel, et de plus ou moins deux fois pour le cobalt et le manganèse.

\section{DISCUSSION ET CONCLUSIONS}

1. Notre étude met en évidence la bonne concordance, constatée ailleurs également dans des conditions d'analyses plus diversifièes, entre les différentes espèces de bryophytes aquatiques prises en considération; celle-ci est d'autant plus satisfaisante que les valeurs observées sont importantes, en particulier pour le cuivre. Une divergence constante est cependant observée entre l'accumulation des métaux lourds par Cinclidotus nigricans et Fontinalis antipyretica à Saint-Valéry, en raison probablement du caractère exceptionnel de cette station soumise régulièrement à l'influence de la mer.

2. La méthode utilisée permet de définir quelques zones de contamination par métaux lourds dans la Somme:

- la région de Ham-Offoy-Epénancourt pour le cuivre, le plomb, le fer, et plus encore pour le nickel, le cobalt et le manganèse ;

- la proximité amont et l'aval d'Amiens pour le nickel, le manganèse, le cobalt, et plus encore pour le fer, le plomb, le chrome, le cadmium et le cuivre.

3. Inversement, la zone comprise entre Frise et Daours semble en général privilégiée, ainsi que la région de Pont-Rémy. Là, une diminution sensible dans la concentration en métaux lourds est régulièrement observée dans toutes les espèces présentes; elle est particulièrement nette pour le cuivre.

4. L'absence de bryophytes aquatiques à Séraucourt nous prive de données qui seraient très intéressantes dans cette zone largement polluée par les métaux lourds (des analyses réalisées dans l'eau à Séraucourt montrent de très fortes concentrations en $\mathrm{Zn}$ et $\mathrm{Ni}$ par exemple). 


\section{TRAVAUX CITES}

EMPAIN A., 1973. - La végétation bryophytique aquatique et subaquatique de la Sambre belge, son déterminisme écologique et ses relations avec la pollution des eaux. Lejeunia, N.S., 69, 58 pp.

EMPAIN A., 1974. - Relations quantitatives entre les bryophytes de la Sambre belge et leur fréquence d'immersion : distribution verticale et influence de la pollution. Bull. Soc. roy. Bot. Belg., 107, 361-374.

EMPAIN A. et LAMBINON J., 1975. - Les bryophytes aquatiques et subaquatiques en tant que bioindicateurs de la pollution des eaux douces. Bull. Soc. Bot. Fr., 121 (Colloque Lille 1972), 257-264.

KIRCHMANN R. et LAMBINON J., 1973. - Bioindicateurs végétaux de la contamination d'un cours d'eau par les effluents d'une Centrale nucléaire à eau pressurisée; évaluation des rejets de la Centrale de la SENA (Chooz, Ardennes françaises) au moyen des végètaux aquatiques et ripicoles de la Meuse. Bull. Soc. roy. Bot. Belg., 106, 187-201.

FINTA M., 1971. - Spectrométrie d'absorption atomique, Tome II, Application à l'analyse chimique. Paris, Masson, 287-793 + I- XXIX. 